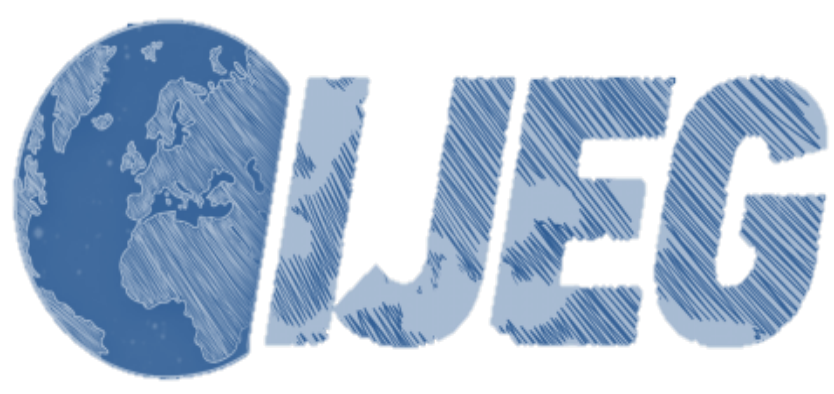

International Journal of Engineering and Geosciences (IJEG),

Vol; 3; Issue; 3, pp. 108-118, October, 2018, ISSN 2548-0960, Turkey,

DOI: $10.26833 /$ ijeg. 413473

\title{
POSSIBLE CONTRIBUTIONS OF SPATIAL SEMANTIC METHODS AND TECHNOLOGIES TO MULTI-REPRESENTATION SPATIAL DATABASE PARADIGM
}

\author{
Memduhoglu, A. ${ }^{1 *}$, Basaraner, M. ${ }^{2}$ \\ ${ }^{1}$ Harran University, Department of Geomatic Engineering, Division of Cartography, Sanliurfa, Turkey \\ (akadirm@harran.edu.tr); \\ ${ }^{2}$ Yildiz Technical University, Department of Geomatic Engineering, Division of Cartography, Istanbul, Turkey \\ (mbasaran@yildiz.edu.tr);
}

ORCID ID 0000-0002-9072-869X; ORCID ID 0000-0002-4619-7801

*Corresponding Author, Received: 07/04/2018, Accepted: 19/04/2018

\begin{abstract}
Today, the amount and variety of spatial data have increased dramatically. In addition, the web has made it easier to disseminate and share this kind of data. Therefore, spatial data integration and interoperability have gained more importance. Spatial data are collected from different sources and often heterogeneous in terms of the levels of detail and the points of view. To able to meet the demands of different spatial applications, multi-source and heterogeneous spatial datasets need to be integrated as well as the consistency of these datasets needs to be maintained. In this context, multirepresentation spatial database (MRSDB) paradigm has been suggested by researchers. However, the heterogeneity constitutes a significant barrier in this respect and hence the implementations have so far been remained within a rather narrow scope. In this article, it is mainly discussed about the possible contributions of basic methods and technologies of spatial semantics such as ontologies, semantic web and linked data to the data integration for creating a MRSBD. Some examples are also given to illustrate the concept.
\end{abstract}

Keywords: Spatial Semantics, Multi-Representation Spatial Databases, Semantic Interoperability, Spatial Ontologies, Linked Spatial Data 


\section{INTRODUCTION}

In recent years, along with the progress in spatial data acquisition technologies such as sensors and global navigation satellite systems, large amounts of spatial data have been produced. This has led to the emergence of many forms of the same spatial phenomenon in different spatial datasets. A spatial phenomenon can be represented in various ways from semantic, geometric and/or graphic aspects depending on specific application requirements (Friis-Christensen et al., 2005; Basaraner, 2012). In this context, multi-representation spatial database (MRSDB) has emerged as an important paradigm that allows spatial data about the same real world phenomenon coming from various sources to be kept at different levels of detail integrally. For this purpose, spatial data integration problem needs to be solved so that heterogeneous spatial data belonging to a same region or same real world phenomena can be linked and used together. The integration problem is more than a syntactic one. Therefore, it is not only be solved by adherence to one standard file format such as Geography Markup Language (GML) but also involves understanding the meaning of data. Applying semantic web technologies can be a promising approach for reducing the cost and improving the accuracy of the integration by making semantic differences among spatial datasets explicit in ontologies (Hart et al., 2013). Therefore, when used in conjunction with semantic web technologies, the MRSDB paradigm can be used for analysis and visualization at multiple levels of detail and, when necessary, for automatic transfer of updates from finer to coarser levels of detail. Automatic updates between levels of detail are also important for ensuring consistency between datasets of different resolutions (Wang et al., 2009).

National Mapping Agencies (NMAs) such as the Ordnance Survey (OS) of Great Britain and the United States Geological Survey (USGS), which are responsible for production, management and dissemination of spatial data in order to meet the various needs of the relevant institutions and organizations, are working for publishing their data as linked data with semantic web technologies. In this context, taxonomies and geographical (geo-) ontologies for various domains have been produced by these NMAs (Goodwin et al., 2008; Varanka, 2009; Varanka et al., 2015). Ontology is the conceptualization and modeling of classes, entities, and relations of a specific domain with the help of logic systems (Guarino, 1998; Uitermark, 1998; Thomson, 2009; Memduhoglu et al. 2017). On the other hand, a geo-ontology does not consist of only semantic relations such as synonymy, similarity, mereonomy and hyponymy, but also spatial relations such as adjacency, containment and connectedness (Fonseca et al., 2009).

The development and maintenance of ontologies are important to utilize existing data for different purposes. In this regard, ontology integration is a crucial issue for reuse and share information among different communities and to create a knowledge base about a domain (Kavouras et al., 2007). Although there are different classifications in the literature, three levels of ontology can be mentioned in the most general sense: high-level (global) ontologies, domain (task) ontologies and application ontologies (Guarino, 1997). Microontologies have been added to this classification at the bottom level by Hart et al. (2013). Recently, ontology design pattern approach, which comprises special design steps for various applications, is also used as application ontologies (Carral et al., 2013; Hu, 2017). Ontology design pattern is defined by Sinha et al. (2014) as small ontologies capturing essential and reusable characteristics of a certain domain. Geo-ontologies or ontologies that belong to the spatial domain usually are domain ontologies.

A MRSDB stores various connected spatial datasets at different levels of detail (resolution/scale) about a geographic space in order to create a more sophisticated and flexible environment not only for multi-purpose geographic data and map production but also for multilevel spatial analysis and visualization. In other words, MRSDB paradigm simplifies the costly and time consuming tasks of production, management and maintenance of datasets and eliminates inconsistencies among associated datasets.

From a broader perspective, a MRSDB include interlinked topographic and thematic geographic and cartographic datasets at different levels of detail (Basaraner, 2012, 2016). The same real world phenomenon is represented in different feature classes and/or with different semantic, geometric and/or graphic definitions in these datasets because spatial datasets are set up to serve different purposes (Figure 1). To be specific, this heterogeneity mainly results from different semantic, geometric and/or graphic resolutions and different themes such as topography, navigation and tourism about which spatial datasets are created. There are two main methods for creating a MRSDB: (1) generalization and (2) integration. In the first approach, spatial data is generated in highest resolution or at largest scale and then transferred to lower resolutions or smaller scales through generalization. During generalization process, the links between spatial features at different levels of detail are stored. In the second approach, spatial features in separate but associated datasets are interlinked based on semantic and geometric matching rules. Since, generalization is beyond the scope of this paper, creating MRSDBs through integration is discussed here.

Despite the advantages mentioned above, it is not so easy to handle the inconsistencies between multiple spatial datasets. Due to the lack of sophisticated tools that can find and link corresponding spatial features at those datasets, building a MRSDB is a challenging task.

This study aims at investigating possible contributions of spatial semantic methods and technologies to the creation of MRSDBs. To this end, first heterogeneity issues in spatial data are explained. Then, approaches and challenges about the integration of heterogeneous spatial data in MRDBs as well as the potential role that semantic web methods and technologies play within this scope are addressed.

The rest of the paper is organized as follows: Section 2 discusses heterogeneity problems of spatial data and possible solutions to them. Section 3 goes through the data integration and interoperability in terms of spatial semantics. Section 4 introduces semantic methods and technologies within the context of spatial domain. Section 5 describes the potential use of spatial semantic methods for MRSDBs and gives a short overview on current approaches as well as presents simple ontologies as a conceptual use case. Finally, Section 6 concludes the paper. 


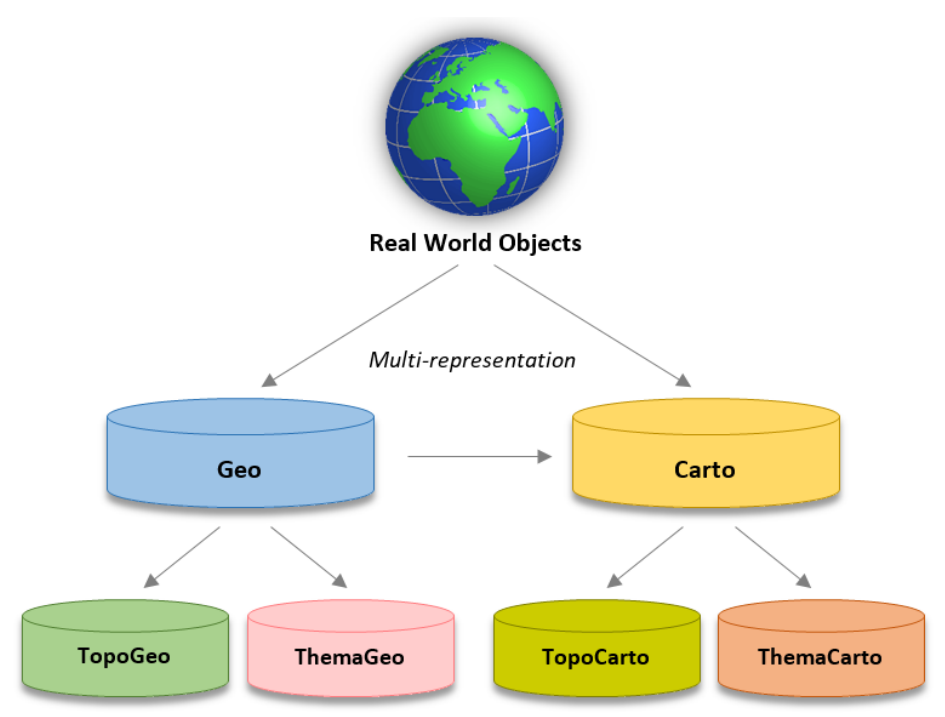

Figure 1. From real world objects to topographic and thematic geographic and cartographic datasets in MRSDB (adapted from Basaraner, 2016)

\section{SPATIAL DATA HETEROGENEITY}

By its nature as an interdisciplinary area, geographic information science deals with spatial heterogeneity problems. In this section, this kind of heterogeneity problems are investigated and possible solutions to them are discussed along with the literature review.

One of the first categorization about the spatial heterogeneity is provided by Bishr et al. (1999). They divides the heterogeneity into three categories: semantic, schematic and syntactic. Hakimpour (2003), Euzenat et al. (2007) and Khatami et al. (2010) also mention about spatial heterogeneity problems and make similar categorizations to the those proposed by Bishr et al. (1999). Brodeur (2012) and Brodaric (2017) add another level called system to this categorization which specifies heterogeneity between systems. Delgado et al. (2013) makes a classification about spatial heterogeneity types which can occur during ontology integration. These categories are: terminological, syntactic and conceptual heterogeneities. Volz (2005) divides database integration into two steps: schema integration and object (or data) integration. Hess et al. (2007) also categorize heterogeneities as concept-level and instance-level similar to the those proposed by Volz (2005).

Syntactic and structural heterogeneity can occur between two datasets at the technical level. Syntactic heterogeneity concerns the physical representation of data while structural heterogeneity refers to differences in data modeling (Brodeur, 2012). For instance, syntactic heterogeneity can occur when two datasets defined in different spatial formats or encodings while structural heterogeneity can occur when data defined with two different geometry type or concept. On the other hand, semantic heterogeneity can occur at the conceptual level (e.g. two different representations of the same object at different levels of detail or at different points of view).

Syntactic and structural heterogeneity problems can be eliminated with standards and specifications. By using XML (eXtensible Markup Languge), GML (Geography
Markup Language), RDF (Resource Description Framework), and OWL (Web Ontology Language) these two types of problems can be solved. Communication between different systems can be ensured by protocols such as Ethernet, Transmission Control Protocol/ Internet Protocol (TCP/IP) and Hypertext Transfer Protocol (HTTP). The use of these protocols also removes heterogeneity problems of systems at the same time (Brodeur, 2012; Varol et al., 2017). But semantic heterogeneity problem needs more complex solutions. Using ontologies at modelling of spatial data and matching these ontologies with either manual or more ideally with automatic techniques is the most promising approach for solution of semantic heterogeneity problem.

One of the factors that complicate the harmonization of spatial datasets is the problem of multilingualism (Annoni et al., 2008). Looking at projects such as Infrastructure for Spatial Information in Europe (INSPIRE), one of the major problems that the responsible parties are confronted is that the multilingual inconsistencies result from a multinational structure. Ontology matching can be a potential solution for that problem too. For example, Figure 2 illustrates the land use classifications of Austria and European Coordination of Information on the Environment (CORINE). In the classifications, the corresponding classes at the higher level can be matched easily because there is not a big conflict (naming conflict) but the situation becomes complicated when shifting to a lower level, and matching becomes difficult. This difficulty can be solved by matching techniques and algorithms which will be discussed at the following sections. As taxonomies are seen as the first step in creating ontology, it is possible to match large ontological spatial data such as national spatial data infrastructures in a similar way (Cömert et al., 2008; Ulutas et al., 2016). The matching process can also eliminate the multilingual problem as the classification used in semantic definitions or ontology is done partly independently from the language. 


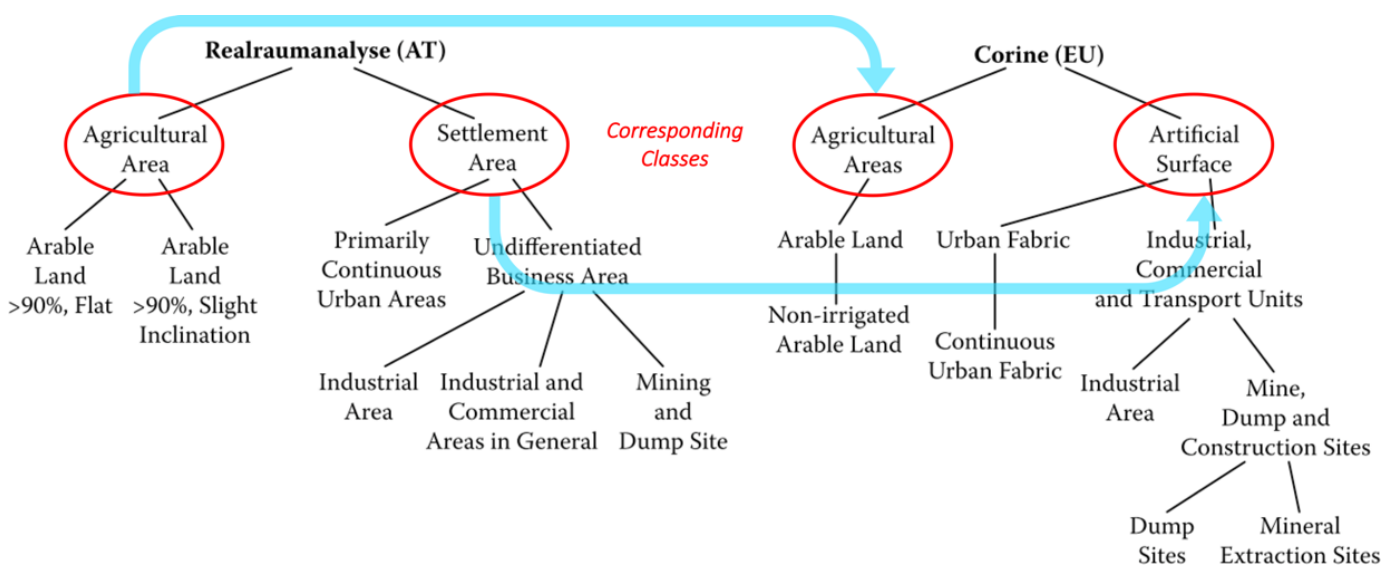

Figure 2. Example of land use classification systems of the Austrian Realraumanalyse (left) and the European CORINE (Annoni et al., 2008)

\section{SPATIAL DATA INTEGRATION AND INTEROPERABILITY}

The fusion and integration of spatial data is one of the hot topics that are still being discussed in the geographic information community (Yi, 2013). In order to be able to use, analyze or visualize the data of the same real world object from different sources, produced at different levels of detail and/or from different points of view, it is necessary to integrate the datasets by eliminating heterogeneity problems (Figure 3). Semantic web and related technologies have the potential for a solution to the existing integration problem.

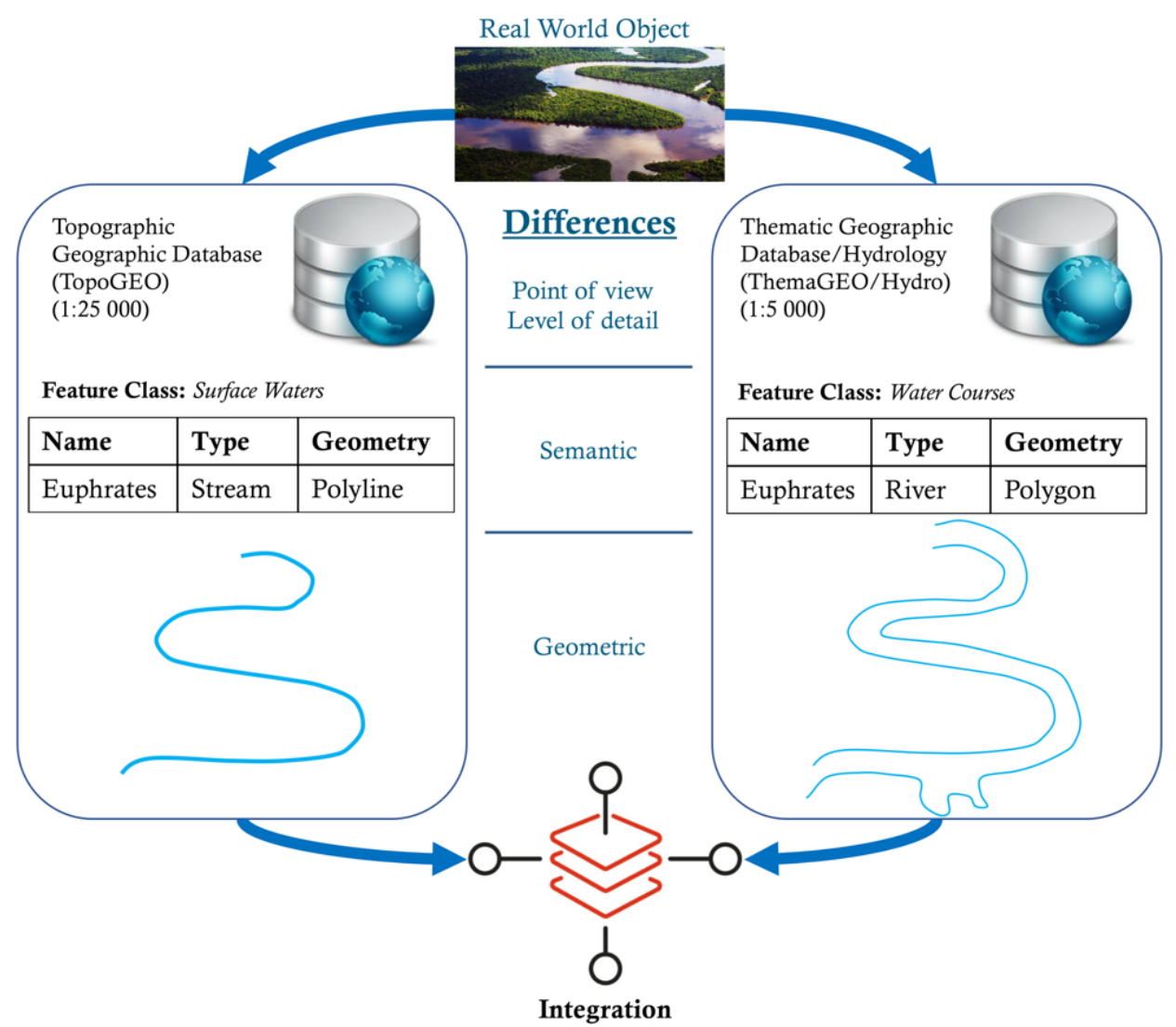

Figure 3. Heterogeneous real world objects in terms of point of view and level of detail which affect their semantic and geometric definitions.

The ontology that provides the conceptualization of the application domain and the knowledge representation provides a potential and support for the integration of heterogeneous information coming from multiple sources and the matching of entities (Rodríguez et al., 2003; Stoter et al., 2006). Since it is not possible to use a single ontology for multiple themes, different level of details or different languages, more than one ontologies needed in 
most cases. Using these multiple ontologies together and relating them to one another can support heterogeneous information systems (Kieler, 2008; Stock et al., 2011). With the integration, it is possible to obtain a third stronger dataset by combining two datasets that have strong sides on different parts. For example, a dataset obtained from the integration of two datasets with more detailed attributes and more detailed geometry will be able to respond to more complex questions within better accuracy (Uitermark et al., 2005). A benefit of obtaining stronger datasets by aligning different sources of information is to unveil knowledge with automatic machine inference that cannot be discovered easily by humans (Hahmann et al., 2010). Currently there are two main methods to relate ontologies with each other: first one is ontology merging which is creation of global vocabulary/ontology by merging ontologies to map local terms in each dataset and the other one is ontology alignment defined as computation of similarity between local entities and create relations between them (Stock et al., 2011; Wiegand et al. 2015).

In the field of ontology matching, there are some close terminologies that can lead to misuse. These are: mapping, integration, alignment, merging and matching. They are all applied to heterogeneous ontologies, but they have some differences. Clarifying them will prevent their misuse. Ontology mapping is finding relations between similar instances or concepts based on statistics. It maintains a link between two ontologies. Ontology integration takes two ontologies as input and gives an adjusted ontology. Ontology alignment is set of corresponding concepts between ontologies and also it shows degree of similarity. It keeps consistency of original ontology. Ontology merging is developing a new sophisticated ontology based on two or more ontologies. Lastly the ontology matching is a set of correspondences itself. Ontology matching is similar to the ontology alignment approach but difference is that matching involves correspondences rather than concepts (Kipngenoh, 2010).

Despite the advantages mentioned above, the use of ontologies for integration has some drawbacks as well. These are: (1) expensiveness of time, complexity and quality of building ontologies and (2) difficulty of proper formalisms to support by reasoning systems (Hakimpour, 2003).

\section{SPATIAL SEMANTIC METHODS AND TECHNOLOGIES}

The semantic web concept was first introduced by Tim Berners-Lee in 2001, which is known as the founder of the web (Berners-Lee et al., 2001). Semantic web emerged as an extension of the existing web that allows people and machines to work together provided with a well-defined meaning of information, allows web to exist not only for the understanding of humans but also for machines (Lassila et al., 1999). With logic systems (description logic, first order logic etc.), this concept provides a potential for making meaningful information from big data concept which is the collection of divergent data from everywhere.

Resource Description Framework (RDF), which is a building block at the heart of the semantic web and its applications, allows the definition of any web resource to be interpreted by the relevant machine (Beckett et al., 2004; Sikos, 2015). RDF is a semantic data modeling language in which data is held in the form of subject, object and predicate. For example, when said "Ankara is the capital of Turkey, Ankara is the subject, Turkey is the object and capital is the predicate which expressing the relationship between the subject and the object (Figure 4). When these expressions are defined with semantic web technologies together with specific vocabularies and rules, the machines can infer meaningful information from these expressions. When looking at the example mentioned above, machines can infer that Ankara is a city, Turkey is a country and every country has only one capital so Istanbul is not the capital of Turkey (van Harmelen, 2008). When these RDFs get related or connected with each other, they become linked data (Hahmann et al., 2010). The semantic web approach, where linked data is embedded, can transform the classical web phenomenon that exists only in the form of documents which only people can understand and interpret into a case where also machines can automatically inference and make Berners-Lee's vision possible (Hu et al., 2016). The LinkedGeoData initiative, which transforms OpenStreetMap to the RDF knowledge base with linked data rules and links this data to other knowledge bases such as DBpedia and Geo Names, contributing to the geospatial part of the semantic web (Auer et al., 2009).

As an extension of RDF, Web Ontology Language (OWL) can be employed for defining the advanced relationships between concepts. Although OWL is based on RDF, more advanced class definitions and restrictions can be made with OWL. For example, we can express Ankara is the only capital of Turkey and no other city can be capital with OWL's inverseFunctionalProperty feature. OWL uses description logic (DL) and makes decisions based on this mathematical model. The documents created with the OWL are also known as ontologies that can be published over the Web. OWL has three sublanguages: OWL Lite, OWL DL, and OWL Full. OWL Lite provides language constructs for a classification hierarchy and simple constraints. OWL DL supports the maximum expressiveness while retaining computational completeness and decidability. OWL Full provides the maximum expressiveness and more syntactic freedom for RDF with no computational guarantees. As understood from the definition, OWL DL is the most common sublanguage for the creation of ontologies. The simplest form of the language can be defined as the concepts such as classes, properties, and individuals (Figure 5). 


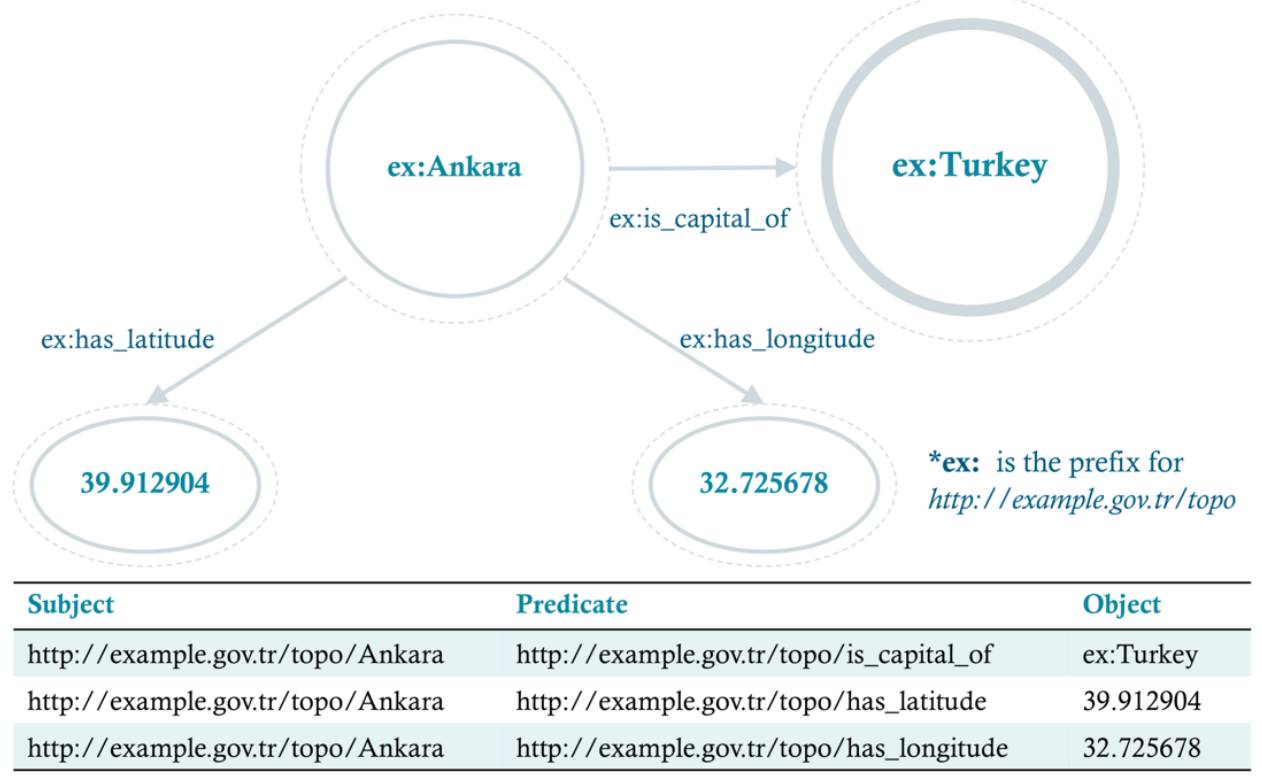

Figure 4. The RDF structure in the form of triples (adapted from Memduhoglu et al., 2017)

Class is a set of individuals that share common properties. Properties define relationships between individuals or data values. Individuals are instances of classes and properties. For instance, as seen in the Figure 5, England, Turkey and USA are instances of country class and livesInCountry corresponds to the property that expresses relationship. OWL is a declarative language which represents knowledge rather than programming language. The set of classes and statements about classes is known as the Terminological Box or Tbox. The set of Individuals and facts about them is known as the Assertion Box or Abox. It is possible to define and automate the use of the ontologies in data integration through OWL, which are made up of a large number of systems with their own semantic meanings. Using these diverse systems together in a common ontology, OWL supports spatial information interoperability at the semantic level. The query language of semantic web is SPARQL (SPARQL Protocol and RDF Query Language) developed by the World Wide Web Consortium (W3C). However, SPARQL does not support spatial queries and analysis. To query semantically defined data and perform basic spatial analysis, GeoSPARQL standard has been developed by OGC as an extension to the SPARQL standard (McGuinness et al., 2004; Prud'hommeaux et al., 2008; Perry et al., 2012; Hart et al., 2013; Zhang et al., 2015).

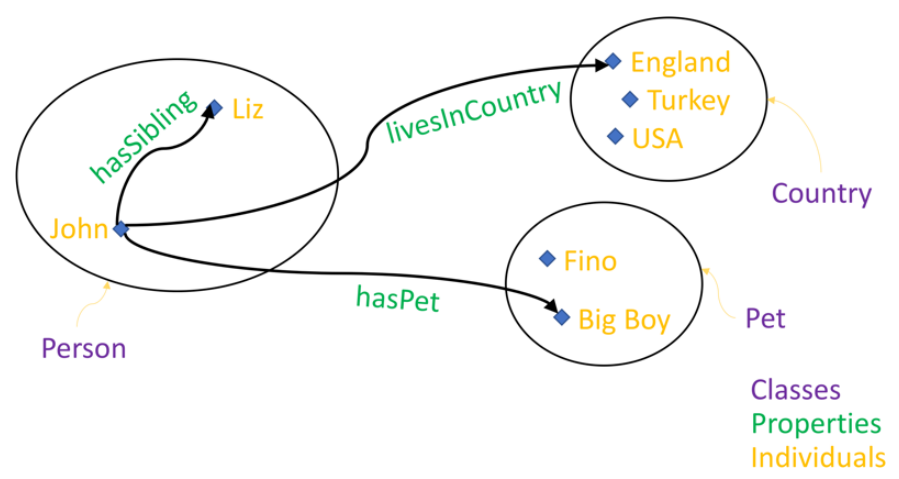

Figure 5. The components of OWL which consist of Classes, Properties and Individuals (adapted from Horridge et al., 2013)

Protégé is a commonly used open source software for building and using ontologies. Protégé supports OWL and RDF ontologies to be loaded, edited and visualized. It also allows importing Semantic Web Rule Language (SWRL) files. Protégé contains various plugins and reasoners like HermiT, $\mathrm{FaCT}++$ and Pellet. With these reasoners the knowledge can be inferred from data which exist in the form of ontologies. Protégé has also a web version called WebProtege. Projects can be loaded into the WebProtege and used ubiquitously. There is a commercial alternative to the Protégé called TopBraid Composer which is also an ontology editor and a visual modeling environment for creating and managing ontologies in the semantic web standards RDF and OWL. In addition to the ontology editors there are plenty of tools, libraries and software packages to support semantic web. For instance, Jena is a free and open source Java development platform for the semantic web applications. Jena contains the following components: Fuseki which is SPARQL server and TDB which is triple store that stores 
RDFs. Since aforementioned software are all open source, they have strong community support and wellstructured documents that can be found freely on the web.

There are also semantic matching/alignment tools and algorithms worth to mention. Ontology Alignment Evaluation Initiative (OAEI) has the goal for evaluation of the increasing number of methods available for schema or ontology matching. For this goal, the initiative also organizes an annual evaluation event and publishes the tests and results of the event for further analysis. One of these tools is S-Match, which is an open source semantic matching framework that provides several semantic matching algorithms and facilities for developing new ones. The other tools are Alignment API, AROMA, BLOOMS and RIMOM. (Delgado et al., 2013; OAEI, 2018)

GML is an Open Geospatial Consortium (OGC) and International Organization for Standardization (ISO) encoding standard for the representation, storage and exchange of geographical features to ensure interoperability. In general, GML is a XML implementation to describe generic geographic data sets that contains points, lines and polygons. Since GML does not define geographic objects, it can be extended to define core reference ontologies or schemas like CityGML. Although current version (2.0) of CityGML does not support multi representation of features, the upcoming version (3.0) will support the multi representation framework. In this context, Löwner et al. (2016) discuss alternative profiles for new version of CityGML to support multi representation framework (Cömert et al., 2010; Roussey et al., 2011; CityGML, 2018)

GML can help to resolve structural and syntactic heterogeneity problems but cannot resolve semantic heterogeneity problems directly. Instead of this, as a standard representation of geographic features, GML can support the translation of geographic features into machine understandable forms like RDF or OWL. Since, GML is widely adopted by geospatial community and it is commonly used in spatial data infrastructures, the web of linked data could profit from this effort, as large amounts of standardized spatial information could be made available as linked data. (van den Brink et al., 2014; Zhang et al., 2015)

Although, RDF and OWL are good alternatives for GI community to adopt the modern methods and techniques that suit to information age, they have a significant limitation in terms of spatial indexing and performing advanced GIS operations (Goodwin et al, 2008).

\section{USE OF SPATIAL SEMANTIC METHODS AND TECHNOLOGIES FOR MRSDB}

MRSDB provides an efficient environment for multipurpose spatial data modeling, processing, analysis, visualization and dissemination. On the other hand, semantic web methods and technologies are promising for automatic integration and inference. In this sense, combining two approaches will provide the best of both worlds.

In the literature, a few approaches are proposed toward MRSDB. Among them, some approaches are potentially more pertinent. The first one is the VUEL approach, which is described by the author as "a unique combination of visible elements (geometry and graphic symbols) with a particular semantics". The VUEL uses Unified Modelling Language (UML) notation to present table linking three dimensions: geometric, semantic and graphic (Bedard et al., 2002).

Another approach is the stamping technique which can be considered more relevant to semantic web technologies (Vangenot et al., 2002). The stamping technique takes the form of binaries to represent object from two perspectives. From these two perspectives, the first one stands for the point of view and the latter represents the level of detail or resolution. Each of these binary representations is called a stamp, for instance ("cartography", 1 meter) and stamps characterize different representation of real world objects. In this regard, two approaches are proposed for multi representation: the integrated approach where the stamping technique is used to define customized data structures and the inter-relationship approach where representations are linked through correspondence links.

Benslimane et al. (2003) take the stamping technique one step further and merged the stamping technique with the description logic to create a DL-based ontology language for multi-representation. They create set of constructors based on DL and stamping technique and present a contextual ontology language to support multi representation ontologies called MuRO (MultiRepresentation Ontologies). MuRO is an ontology which expresses a specific domain by a variable set of properties or attributes in several contexts.

Friis-Christensen et al. (2005) develop a Multi Representation Management System (MRMS) to manage and model multiple representations. They present a Multiple Representation Schema Language (MRSL) which is a conceptual schema language used to describe consistency requirements in a multiple representation context within spatial data. The basic elements of the MRSL are matching, consistency and restoration rules. The graphical notation of MRSL is based on an extension to UML and the Object Constraint Language (OCL). They use stereotypes which provide a way of defining a virtual subclass of a UML metaclass with additional semantics.

A more recent study about this topic has been realized by Stoter et al. (2011). They present an information model for a multi-scale topographic database containing rich semantics both on multi-scale data content and on scale transitions. Information Model Topography (IMTOP) model has been expressed using the UML and complemented with OCL. The IMTOP uses inheritance, derivation relationships and derived attributes to model a class at specific scales. The advantage of this approach is its simplicity at reading and deriving a model per map scale by showing the relevant classes for that map scale only and the disadvantage is that it is expressed with UML and OCL instead of RDF or OWL. The authors also state that transferring IMTOP into an ontology language such as RDF or OWL will make more semantics known at machine level required for wide reuse of the data.

The aforementioned studies, methodologies and tools about the MRSDB have not gained mainstream adoption owing to the complexity of the process of creating MRSBDs. The lack of software tools that can provide the definition of OCL expressions and the use of confusing schemas involving very long OCL expressions make it difficult to use widely. Using these techniques with OWL or RDF will provide them a powerful background of 
standards and ease of the adoption from geographic information community. Furthermore, the application of a description logic language like OWL will allow inferences from datasets in a MRSDB.

Creating taxonomies is an important step to build ontologies and provide interoperability of spatial information. As an example, three groups of simple ontologies created from taxonomies at three different levels of detail (LoD) were presented in Figure $6 \mathrm{a}, 6 \mathrm{~b}$ and 6c. Following ontologies were created: (1) topographic geographic ontology (TopoGEO) which corresponds to topographic point of view for a group of feature classes such as hotels, shops and restaurants (Figure 6a), (2) thematic geographic ontology for tourists (ThemaGEO/Tourist) which is created for the use of tourists (Figure 6b), and (3) thematic geographic ontology for cities (ThemaGEO/City) which corresponds to city managers' point of view (Figure 6c). The heterogeneity problems occur due to the ontologies have different points of view and levels of detail (LoD). For instance, hotel is in: LoD 3 and under Building and Construction $>$ Social Facility class in TopoGEO, LoD 2 and under Accommodation class in ThemaGEO/Tourist, LoD 3 and under Tourism > Accommodation class in ThemaGEO/City. It can be seen that Hotel class is different both in LoDs and classifications. In order to eliminate this heterogeneity problem, matching techniques and algorithms can be applied to the ontologies either manually or automatically. After the matching step an appropriate MRSDB method can be used to achieve the goal. After this last stage, an MRSDB powered with semantics can be established to analyze spatial data through levels of detail and new knowledge can be inferred automatically by machines from existing datasets

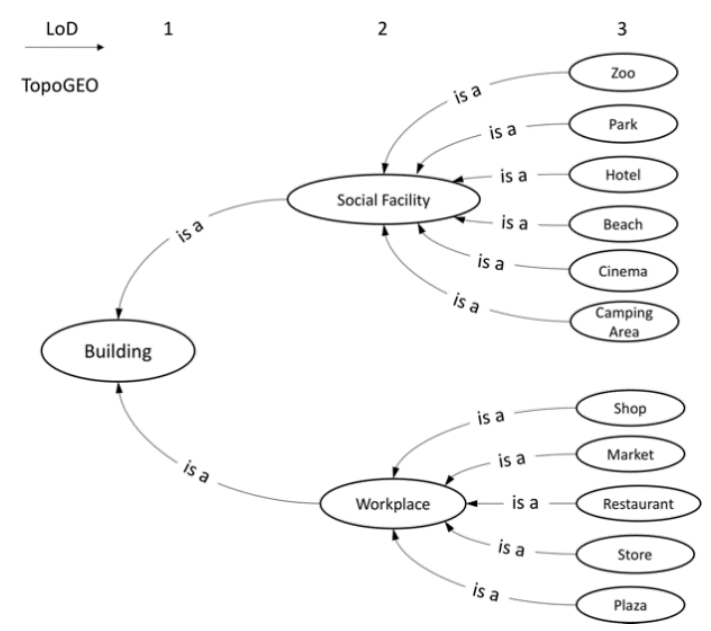

Figure 6a. Topographic geographic ontology (TopoGEO)

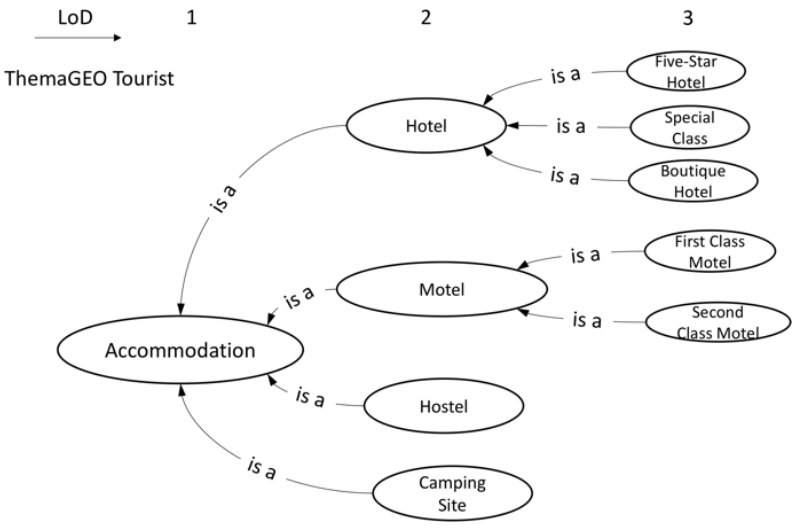

Figure $6 \mathrm{~b}$. Thematic geographic ontology for tourists (ThemaGEO/Tourist)

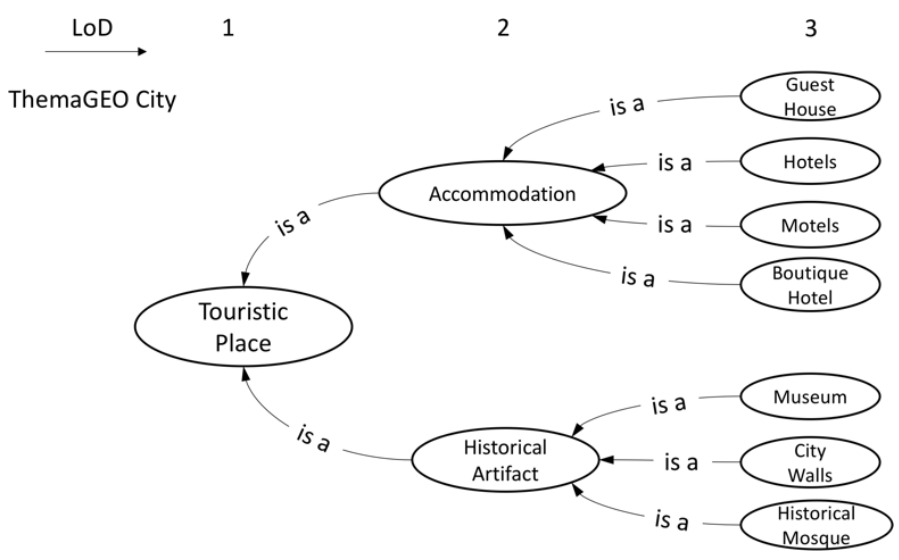

Figure 6c. Thematic geographic ontology for cities (ThemaGEO/City)

\section{CONCLUSION}

In this study, the problems about the integration of spatial data have been identified and it has been investigated whether the methods and technologies about spatial semantics, which appear to be a potential contributor to the solution of these problems, can be utilized for creating MRSDBs. Simple ontologies from three different points of view and three different levels of detail are also created as an example of integration challenge that a MRSDB has to cope with. Incorporating multiple ontologies into MRSDBs as multirepresentations of spatial objects can provide a new insight into the solution of heterogeneity problems and 
the integration of heterogeneous spatial datasets. With such an approach, a MRSDB can be created by means of multiple ontologies, the inconsistencies in the data between different levels of detail can be detected, the semantic integration of various spatial data and multilingual datasets can be ensured and new knowledge inferences can be made (Tanasescu, 2007; Varanka, 2009; Basaraner, 2013; Ulutaş et al., 2016; Hu, 2017; Memduhoglu et al., 2017). Future work will focus on creating ontologies based on RDF/OWL within the scope of MRSDBs.

\section{REFERENCES}

Annoni, A., Friis-Christensen, A., Lucchi, R. and Lutz, M., 2008. Requirements and challenges for building a European spatial information infrastructure: INSPIRE, In Creating Spatial Information Infrastructures: Towards the Spatial Semantic Web (van Oosterom P. and Zlatanova S. Eds.), CRC Press, Boca Raton, Florida, USA.

Auer, S., Lehmann J. and Hellmann S., 2009. Linkedgeodata: Adding a spatial dimension to the web of data, 8th International Semantic Web Conference (ISWC 2009), Washington, DC, USA, pp. 731-746.

Basaraner, M., 2012. An investigation of semantic, geometric and graphic heterogeneities of building and facility objects in a multi-resolution spatial database, Proceedings of 4th International Conference on Cartography and GIS, Albena, Bulgaria.

Basaraner, M., 2013. Taxonomies of building objects towards topographic and thematic geo-ontologies, 26th International Cartographic Conference, Dresden, Germany.

Basaraner, M., 2016. Revisiting cartography: towards identifying and developing a modern and comprehensive framework, Geocarto International, 31(1), 71-91.

Beckett, D. and McBride, B., 2004. RDF/XML syntax specification (revised), W3C Recommendation, W3C Consortium.

Bedard, Y. and Bernier E., 2002. Supporting multiple representations with spatial databases views management and the concept of VUEL, ISPRS/ICA Joint Workshop on Multi-Scale Representations of Spatial Data, Ottawa, USA, pp. 1-14.

Benslimane, D., Vangenot C., Roussey C. and Arara A., 2003. Multirepresentation in ontologies, Proceedings of Advances in Databases and Information Systems: 7th East European Conference (ADBIS 2003) (Kalinichenko, L., Manthey, R., Thalheim, B. and Wloka, U., Eds.), Heidelberg: Springer-Verlag, Dresden, Germany.

Berners-Lee, T., Hendler J. and Lassila O., 2001. The semantic Web, Scientific American, 284(5), 28-37.

Bishr, A. Y., Pundt H., Kuhn W. and Radwan, M., 1999. Probing the concept of information communities-a first step toward semantic interoperability, in Interoperating geographic information systems Springer, Boston, MA, pp. 55-69.
Brodaric, B., 2017. Interoperability of Representations, in International Encyclopedia of Geography: People, the Earth, Environment and Technology (Richardson, D., Castree, N., Goodchild, M. F., Kobayashi, A., Liu, W. and Marston, R. A., Eds.) Oxford, UK: John Wiley \& Sons, Ltd., pp. 1-18.

Brodeur, J., 2012. Geosemantic interoperability and the geospatial semantic web, In Springer Handbook of Geographic Information (Kresse, W. and Danko, D. M., Eds.), Springer, Berlin, Heidelberg, pp. 589-611.

Carral, D., Scheider, S., Janowicz, K., Vardeman, C., Krisnadhi, A. A. and Hitzler, P., 2013. An ontology design pattern for cartographic map scaling, 10th International Conference Proceedings, The Semantic Web: Semantics and Big Data (ESWC 2013) (Cimiano, P., Corcho, O., Presutti, V., Hollink, L. and Rudolph, S., Eds.), Heidelberg: Springer-Verlag, Montpellier, France.

CityGML, 2018. CityGML v3.0 homepage, https://www.citygml.org/ongoingdev/v3/, accessed on 16 March 2018.

Cömert, Ç., Akinci, H., Ulutaş, D., Kara, G. and Yılmaz, C., 2008. Implementing Spatial Data Infrastructures with Semantic Web Services, INSPIRE Conference 2008, Maribor, Slovenia.

Cömert, Ç., Ulutaș, D., Akinci, H. and Kara, G., 2010. Semantic web services for implementing national spatial data infrastructures, Scientific Research and Essays, 5(7), 685-692.

Delgado, F., Martínez-González, M. M. and Finat, J., 2013. An evaluation of ontology matching techniques on geospatial ontologies, International Journal of Geographical Information Science, 27, 2279-2301.

Euzenat, J. and Shvaiko, P., 2007. Ontology Matching, Springer, Berlin.

Fonseca, F. T. and Câmara, G., 2009. Geo-Ontologies, In The GIS Manual (Madden M., Ed.), ASPRS, Bethesda, Maryland, USA.

Friis-Christensen, A., Jensen, C. S., Nytun, J. P. and Skogan, D., 2005. A conceptual schema language for the management of multiple representations of geographic entities, Transactions in GIS, 9(3), 345-380.

Goodwin, J., Dolbear, C. and Hart, G., 2008. Geographical linked data: the administrative geography of Great Britain on the semantic web, Transactions in GIS, 12 (Suppl. 1), 19-30.

Guarino, N., 1997. Semantic matching: formal ontological distinctions for information organization, extraction, and integration, In Information Extraction A Multidisciplinary Approach to an Emerging Information Technology (Pazienza, M. T., Ed.), Springer, pp. 139-70.

Guarino, N., 1998. Formal ontology and information systems, In Guarino, N. (Ed.) Formal Ontology and Information Systems, Amsterdam, IOS Press, pp. 3-15. 
Hahmann, S. and Burghardt, D., 2010. Linked data - a multiple representation database at web scale?, 13th Workshop of the ICA commission on Generalisation and Multiple Representation, Zürich, Switzerland.

Hakimpour, F., 2003. Using Ontologies to Resolve Semantic Heterogeneity for Integrating Spatial Database Schemata, PhD Thesis, University of Zurich, Switzerland.

Hart, G. and Dolbear, C., 2013. Linked Data: A Geographic Perspective, CRC Press, Boca Raton, Florida, USA.

Hess, G. N., Iochpe, C. and Castano, S., 2007. Geographic ontology matching with IG-MATCH, Lecture Notes in Computer Science (Including Subseries Lecture Notes in Artificial Intelligence and Lecture Notes in Bioinformatics), 4605 LNCS, 185-202.

Horridge, M. and Brandt, S., 2011. A practical guide to building OWL ontologies using Protégé 4 and CO-ODE tools, edition 1.3, University of Manchester.

Hu, Y., 2017. Geospatial semantics, In Comprehensive Geographic Information Systems (Huang, B., Ed.), Elsevier, Oxford, UK.

Hu, Y. and Janowicz, K., 2016. Enriching top-down geoontologies using bottom-up knowledge mined from linked data, In Advancing Geographic Information Science: The Past and Next Twenty Years (Onsrud, H. and Kuhn, W., Eds.), GSDI Association Press, Needham, Massachusetts, USA.

Kavouras, M. and Kokla, M., 2007. Theories of Geographic Concepts: Ontological Approaches to Semantic Integration, CRC Press, Boca Raton, Florida, USA.

Khatami, R., Alesheikh, A. and Hamrah, M., 2010. A mixed approach for automated spatial ontology alignment Journal of Spatial Science, 55 (2): 237-255.

Kieler, B., 2008. Semantic data integration across different scales: automatic learning generalization rules, International Archives of Photogrammetry, Remote Sensing and Spatial Information Sciences, 37, 685-690.

Kipngenoh, K. W., 2010. Ontology Mapping for Geoinformation Integration, MSc Thesis, International Institute for Geo-information Science and Earth Observation (ITC), Netherlands.

Lassila, O. and Swick, R. R., 1999. Resource description framework (RDF) model and syntax specification, W3C Recommendation, W3C Consortium.

Löwner, M.-O., Gröger, G., Benner, J., Biljecki, F. and Nagel C., 2016. Proposal for a new LoD and multirepresentation concept for CityGML, ISPRS Annals of Photogrammetry, Remote Sensing and Spatial Information Sciences, IV-2/W1, 3-12.

McGuinness, D. L. and van Harmelen, F., 2004. OWL Web ontology language, W3C Recommendation 10.2004-03.
Memduhoglu, A. and Basaraner, M., 2017. Potential of spatial semantics for developing multi-representation spatial databases, UCTEA International Geographic Information Systems Congress 2017, 15-18 November, Adana, Turkey.

OAEI, 2018. Ontology Alignment Evaluation Initiative, http://oaei.ontologymatching.org/, accessed on 16 March 2018.

Perry, M. and Herring, J., 2012. OGC GeoSPARQL-A geographic query language for RDF data, OGC Implementation Standard.

Prud'hommeaux, E. and Seaborne, A., 2008. SPARQL query language for RDF, W3C Recommendation, W3C Consortium.

Rodríguez, M. A. and Egenhofer, M. J., 2003. Determining semantic similarity among entity classes from different ontologies, IEEE Transactions on Knowledge and Data Engineering, 15(2), 442-456.

Roussey, C., Pinet, F., Kang, M. A. and Corcho, O., 2011. Ontologies for interoperability, in Ontologies in Urban Development Projects (Falquet, G., Métral, C., Teller, J. and Tweed, C., Eds.), Springer, London, pp. 39-53.

Sikos, L. F., (Ed.), 2015. Mastering Structured Data on the Semantic Web, Apress, New York, USA.

Sinha, G., Mark, D. M., Kolas, D., Varanka, D. E., Romero, B. E., Feng, C.-C., Usery, E. L., Liebermann, J. and Sorokine, A., 2014. An ontology design pattern for surface water features, Proceedings of Geographic Information Science: 8th International Conference, GIScience 2014 (Duckham, M., Pebesma, E., Stewart, K. and Frank, A. U., Eds.), Springer International Publishing, Vienna, Austria, pp. 187-203.

Stock, K. and Cialone, C., 2011. An approach to the management of multiple aligned multilingual ontologies for a geospatial earth observation system, in Lecture Notes in Computer Science, GeoS 2011 (Claramunt, C., Levashkin, S. and Bertolotto, M., Eds.), Brest: SpringerVerlag Berlin Heidelberg, Vol. 6631 LNCS, pp. 52-69.

Stoter, J., Lemmens, R., Kobben, B. and Bakker, N. J., 2006. Semantic data integration in a multiple representation environment, ISPRS Workshop on Multiple Representation and Interoperability of Spatial Data, Hannover, Germany, pp. 22-29

Stoter, J., Visser, T., van Oosterom, P., Quak, W. and Bakker, N., 2011. A semantic-rich multi-scale information model for topography, International Journal of Geographical Information Science, 25, 739-763.

Tanasescu, V., 2007. Spatial semantics in difference spaces, proceedings of the International Conference on Spatial Information Theory: Foundations of Geographic Information Science (COSIT) (Winter, S., Duckham, M., Kulik, L. and Kuipers, B., Eds.), Heidelberg: SpringerVerlag, Berlin, Germany. 
Thomson, M.-K., 2009. Dwelling on ontology - semantic reasoning over topographic maps, $\mathrm{PhD}$ Thesis, University of College London (UCL), Retrieved from http://eprints.ucl.ac.uk/17597/

Uitermark, H., Oosterom, P. V., Mars, N., and Molenaar, M., 1998. Propagating updates: Finding corresponding objects in a multi-source environment, In Proceedings of the Eighth Symposium on Spatial Data Handling, Vancouver, Canada, pp. 580-591.

Uitermark, H. T., van Oosterom, P., Mars, N. J. I. and Molenaar, M., 2005. Ontology-based integration of topographic data sets, International Journal of Applied Earth Observation and Geoinformation, 7 (2): 97-106.

Ulutaş, D., Kara, G. and Cömert, Ç., 2016. Semantic definition and matching for implementing national spatial data infrastructures, Journal of Spatial Science, 61(2), 441-459.

van den Brink, L., Janssen, P., Quak, W. and Stoter, J., 2014. Linking spatial data: automated conversion of geoinformation models and GML data to RDF, International Journal of Spatial Data Infrastructures Research, 9, 5985.

van Harmelen, F., 2008. Semantic web technologies as the foundation for the information infrastructure, Creating Spatial Information Infrastructures: Towards the Spatial Semantic Web (van Oosterom, P. and Zlatanova, S., Eds.), CRC Press, Boca Raton, Florida, USA, pp. 3752.

Vangenot, C., Parent, C. and Spaccapietra, S., 2002 Modelling and manipulating multiple representations of spatial data, Advances in Spatial Data Handling: 10th International Symposium on Spatial Data Handling (Richardson, D. E. and van Oosterom, P., Eds.) Heidelberg: Springer-Verlag, Ottawa, Canada, pp. 81-93.

Varanka, D. E., 2009. A topographic feature taxonomy for a U.S. national topographic mapping ontology, Proceedings of the 24th International Cartographic Conference - ICC 2009, Santiago, Chile.

Varanka, D. E. and Usery, E. L., 2015. An applied ontology for semantics associated with surface water features, In Land Use and Land Cover Semantics: Principles, Best Practices, and Prospects (Ahlqvist, O., Varanka, D., Fritz, S. and Janowicz, K., Eds.), CRC Press, Boca Raton, Florida, USA.

Varol, M. B. and Şanlığlu, İ., 2017. Open geospatial consortium web map and feature services and free/open source server/client softwares, International Journal of Engineering and Geosciences, 2(1), 17-26.

Volz, S., 2005. Data-Driven Matching of Geospatial Schemas, International Conference on Spatial Information Theory, Springer Berlin Heidelberg, pp. 115-132.

Wang, Y. H. and Meng, H., 2009. Hierarchical ontology on multi-scale road model for cartographical application, Proceedings - 2009 International Conference on
Environmental Science and Information Application Technology (ESIAT 2009), Wuhan, China, pp. 330-333.

Wiegand, N., Berg-Cross, G. and Zhou, N., 2015. Resolving semantic heterogeneities in land use and land cover, In Land Use and Land Cover Semantics: Principles, Best Practices, and Prospects (Ahlqvist, O., Varanka, D., Fritz, S. and Janowicz, K., Eds.), CRC Press, Boca Raton, Florida, USA.

Yi, S., 2013. Learning ontologies for geographic entity matching and multi-sources data fusion, $21 \mathrm{st}$ International Conference on Geoinformatics, Kaifeng, China, pp. 1-5.

Zhang, C., Zhao, T. and Li, W., 2015. Geospatial Semantic Web, Springer International Publishing, Switzerland.

Copyright (C) International Journal of Engineering and Geosciences (IJEG). All rights reserved, including the making of copies unless permission is obtained from the copyright proprietors. 